This is a postprint version of the following published document:

P.J. Pinzón, I. Pérez, C. Vázquez, J. M. S. Pena (2012)."Reconfigurable $1 \times$ 2 wavelength selective switch using high birefringence nematic liquid crystals". In Applied Optics Volume: 51, Issue:25, Pages: 5960-5965. Available in http://dx.doi.org/10.1364/AO.51.005960

(C) 2012 Optical Society of America 


\title{
Reconfigurable $1 \times 2$ wavelength selective switch using high birefringence nematic liquid crystals
}

\author{
Plinio Jesús Pinzón, ${ }^{*}$ Isabel Pérez, Carmen Vázquez, and José Manuel Sánchez Pena \\ Grupo de Displays y Aplicaciones Fotónicas, Dpto. Tecnología Electrónica, \\ Universidad Carlos III de Madrid, c/ Butarque 15, 28911 Leganés, Spain \\ ${ }^{*}$ Corresponding author: ppinzon@ing.uc3m.es
}

\begin{abstract}
A reconfigurable $1 \times 2$ wavelength selective switch, based on a Lyot filter and high birefringence nematic liquid crystals, is proposed. Simulations and experimental results of a reconfigurable switch are reported. Insertion losses from $3.4 \mathrm{~dB}$ and rejection ratios up to $15 \mathrm{~dB}$ are obtained in a two input channel system at 560 and $621 \mathrm{~nm}$. Control voltages of 0 to 3 VRMS are used.
\end{abstract}

\section{Introduction}

A $1 \times M$ wavelength selective switch (WSS) is an optical device that allows switching any incoming wavelength from its input port to any of the $M$ output ports, without the need of optical to electrical conversions. These devices play a key role in protection and reconfiguration tasks of the next generation optical networks. A number of approaches to implement WSS have been demonstrated. Some are based on gratings that spatially $\mathrm{d}$ isperse $\mathrm{t}$ he input channels, on microelectro-mechan ical systems (MEMS), or on liquid crystal (LC) spatial light modulators [1-4 ]. O ther approa ches use silica-ba sed planar lightwave circuits (PLCs) [5] or ring resonators [6,7].

Many optical filtering and interleaving devices, based on birefringent structures such as the Lyot and Solc filters [8-10] with low dispersion, high reliability, easy manufacturability, and low cost, have been proposed. Those devices usually work in dense wavelength division multiplexing (DWDM) systems, require polarization diversity techniques to avoid polarization dependent loss [11], and do not have reconfigurable features. They usually have the input and one of the outputs in a single port [12], so circulators or other devices are needed to separate both signals.
In future home networks, there is potential for plastic optical fibers (POF) to be used [13,14]. Those networks operate in the visible (VIS) range and they could need reconfiguration because there are no standard channels [15] defined. Some LC reconfigurable devices in VIS have been reported, such as a tunable filter [16] or a multifunctional device operating as a switch, combiner, and variable optical attenuator [17], but not a WSS.

In this work, a reconfigurable $1 \times 2 \mathrm{WSS}$, based on a Lyot filter and high birefringence nematic LCs is proposed. This device is designed to work in the VIS range and uses a simple polarization diversity scheme. Simulations and experimental results, in a two channel system compatible with low cost POF networks, are reported.

\section{Operation Principle and Design}

The proposed reconfigurable WSS is based on tunable Lyot filters with high birefringent nematic LCs. Two different configurations are used for allowing spatial switching of different wavelength channels in a compact design.

\section{A. LC Tunable Lyot Filters}

The Lyot filter $[9,10]$ consist of a series of $\mathrm{N}$ delay stages requiring two linear polarizers $(\mathrm{LPs})$ oriented at $45^{\circ}$ to the optical axis of a retarder bounded (see Fig. 1). 


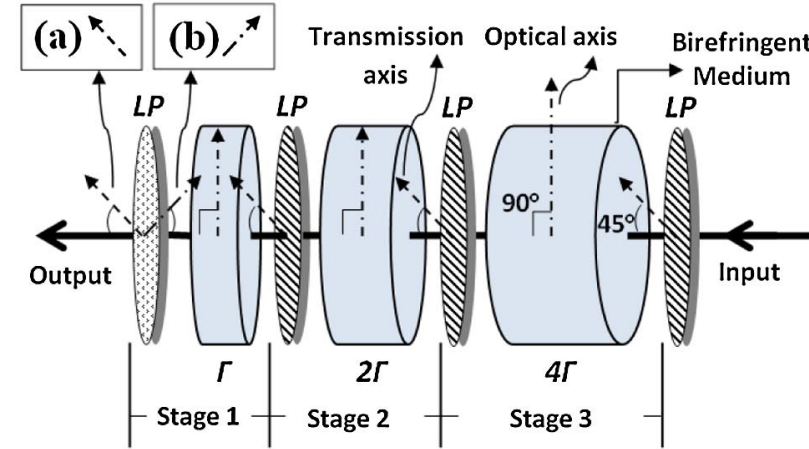

Fig. 1. (Color online) Structure of a 3 Stages Lyot filter with: (a) all linear polarizers, LPs, in parallel; (b) crossed LPs in the stage with lowest delay, $\Gamma$.

Therefore, the transmittance of the $j$ th stage, $T_{j}$, with $j=1,2, \ldots, N$, is a function of the phase delay, $\Gamma_{j}$, between the beam's polarization components passing through the retarder or birefringent medium. This phase delay is given by

$$
\Gamma_{j}=\frac{2 \pi}{\lambda} \Delta n_{j} d_{j},
$$

where $\lambda$ is the wavelength of the input beam, while $\Delta n_{j}$ and $d_{j}$ are the birefringence and thickness of the $j$ th delay stage, respectively. Each stage has twice the delay of the previous one; then $\Gamma_{j}=2^{j-1} \Gamma$ being $\Gamma$ the lowest delay, $\Gamma=\Gamma_{1}$.

In a tunable Lyot filter, each stage is designed with a fixed delay plus a variable delay. In the particular case of an LC tunable filter (LCTF), the variable delay is obtained by using nematic homogeneous liquid crystals (NH-LC). These LC devices have an effective birefringence, $\Delta n_{\mathrm{LC}_{\text {eff }}}$, which is a function of the applied voltage, $V_{\mathrm{LC}}\left(\mathrm{V}_{\mathrm{RMS}}\right)$. If the birefringence of the LC material is high enough, the stages can be designed by only using NH-LC devices.

In a Lyot filter with $N$ stages and parallel LPs [see Fig. 1(a)], the incoming beam can be transmitted in both directions. The transmission, $T_{P}$, is given by

$$
T_{P}=\prod_{j=1}^{N} \cos ^{2}\left(2^{j-1} \frac{\Gamma}{2}\right)=\frac{1}{2^{2 N}} \frac{\sin ^{2}\left(2^{N-1} \Gamma\right)}{\sin ^{2}(\Gamma)} .
$$

$T_{P}$ is a periodic function, and the set of wavelengths with peak transmittance, $\lambda_{P(k)}$, are those having a delay $\Gamma$, which is an integer multiple of $2 \pi$. Those wavelengths are given by

$$
\lambda_{P(k)}=\frac{\Delta n d}{k},
$$

where $k=1,2,3, \ldots$, defines the peak wavelength order. On the other hand, the bandwidth of the transmission band around $\lambda_{P(k)}$ is mainly defined by the number of stages, $N$.

A Lyot filter with crossed polarizers in the lowest delay stage, and parallel polarizers in the other stages, can be implemented by changing orientation of the last polarizer [16]; see Fig. 1(b). In this configuration, the input beam should enter through the stage with the highest delay. The new transmission $T_{C}$ is given by

$$
T_{C}=\sin ^{2}\left(\frac{\Gamma}{2}\right) \prod_{j=2}^{N} \cos ^{2}\left(2^{j-1} \frac{\Gamma}{2}\right)=\frac{1}{2^{2 N}} \frac{\sin ^{2}\left(2^{N-1} \Gamma\right)}{\cos ^{2}(\Gamma)} .
$$

$T_{C}$ is a periodic function, and the set of wavelengths with peak transmittance, $\lambda_{C(m)}$, are those having a delay $\Gamma$, which is an integer multiple of $\pi$. Those wavelengths are given by

$$
\lambda_{C(m)}=\frac{\Delta n d}{m-1 / 2},
$$

where $m=1,2,3, \ldots$, defines the peak wavelength order.

\section{B. Reconfigurable $1 \times 2$ WSS Design}

The proposed reconfigurable $1 \times 2$ WSS is shown in Fig. 2 . Although multiple separation wavelength drops are obtained at the output, there is no influence if only two wavelengths are considered. In this configuration, each arm is based on two Lyot LCTFs but using a polarization beam splitter (PBS) at the lowest delay stage. This PBS acts as both parallel and crossed LPs for each input light polarization state. The input channels are centered at the transmission band of the Lyot filter with parallel and crossed LPs, respectively [see Eqs. (3) and (4)]. The channel centered at the transmission band of the classical Lyot filter [see Eq. (3)] is transmitted at port S1 and the channel centered at the transmission band of the Lyot filter with crossed polarizers [see Eq. (4)] is transmitted at port S2.

Using a scheme with a single arm, input polarizer limits the maximum theoretical transmittance to $50 \%$. This can be overcome by using a polarization diversity scheme, two arms, as in the reconfigurable WSS shown in Fig. 2.

\section{Simulations and Experimental Results}

A reconfigurable $1 \times 2 \mathrm{WSS}$ is simulated and experimentally tested.

The experimental setup is shown in Fig. 3 (just for a single arm of Fig. 2). A halogen light source "Avaligth Hall" and a spectrometer "AvaSpect-128" are used. The fiber optic spectrometer has a 360 to $890 \mathrm{~nm}$ spectral range, and about $4 \mathrm{~nm}$ spectral resolution. Multimode optical fibers and collimators are used at input and output ports.

The LPs have diameter of $12.5 \mathrm{~mm}, 550$ to $1500 \mathrm{~nm}$ operation range, and $70 \%$ polarized light transmission at $600 \mathrm{~nm}$. The PBS is $20 \mathrm{~mm} \times 20 \mathrm{~mm} \times$ $20 \mathrm{~mm}$ and has transmission higher than $90 \%$ in the range from 600 to $700 \mathrm{~nm}$ at both polarizations with a split ratio of unpolarized light of $50 / 50 \pm 5 \%$. 


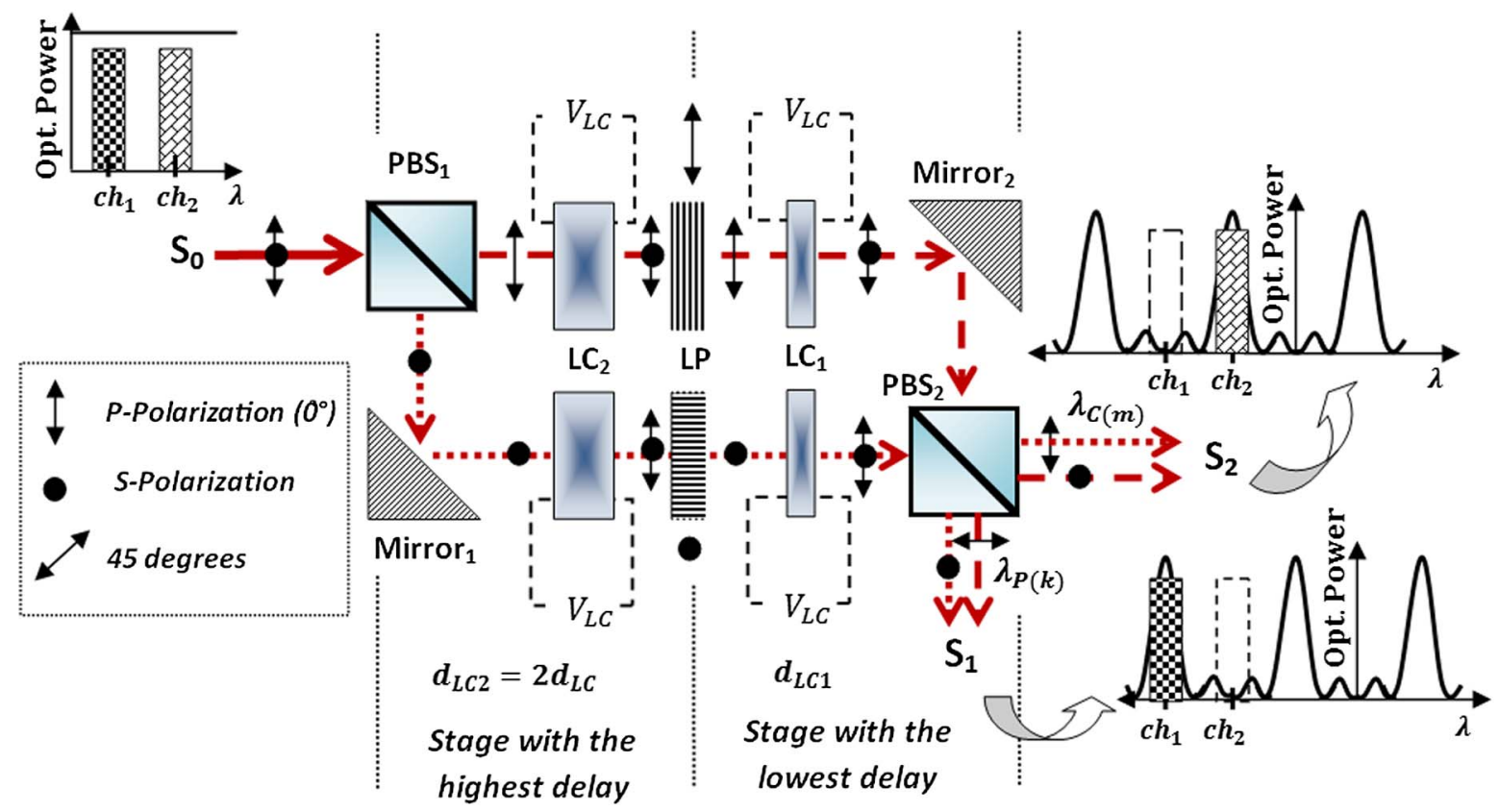

Fig. 2. (Color online) Schematic of a reconfigurable $1 \times 2 \mathrm{WSS}$ with polarization diversity; $d_{\mathrm{LC} 1}$ and $d_{\mathrm{LC} 2}, \mathrm{LC}_{1}$ and $\mathrm{LC}_{2}$ thickness, $V_{\mathrm{LC}}: \mathrm{LC}$ applied voltage.

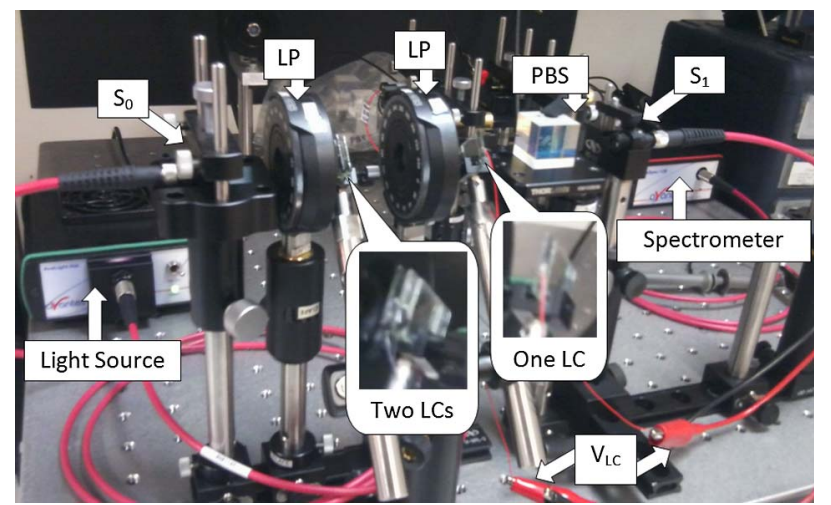

Fig. 3. (Color online) Photograph of the experimental set-up of the reconfigurable $1 \times 2 \mathrm{WSS}$.

Characterization of LPs and PBS is shown in Fig. 4. The NH-LC devices have been specifically designed and manufactured with the mixture 1658, and synthesized at the Military University of Technology in Warsaw. These devices have a thickness $d_{\mathrm{LC}}=$ $5.1 \mu \mathrm{m}$ and birefringence $\Delta n_{\mathrm{LC}}=0.38$ at $633 \mathrm{~nm}$. From Eq. (1) a delay $\Gamma=19.23 \mathrm{rad}$ is obtained. In comparison, the commercially available low birefringence LC mixture MLC-9200-100 $\left(\Delta n_{\mathrm{LC}} \approx 0.11\right.$ at $633 \mathrm{~nm}$ ) produces the same delay with a thickness of $17.6 \mu \mathrm{m}$ [ 18,19]. Thicker LC cells have higher transmission loss, response time, and driving voltage, which deteriorate the overall device performance. The 1658 mixture has a $\Delta n_{\mathrm{LC}}$ high enough to have a good performance in the VIS range, allowing a $1 \times 2$ WSS design only based on NH-LC devices, with $d=d_{\mathrm{LC}}$ and $\Delta n=\Delta n_{\mathrm{LC}}$ eff . Then, to fulfill the design condition, $\Gamma_{j}=2^{j-1} \Gamma$, one LC device is used

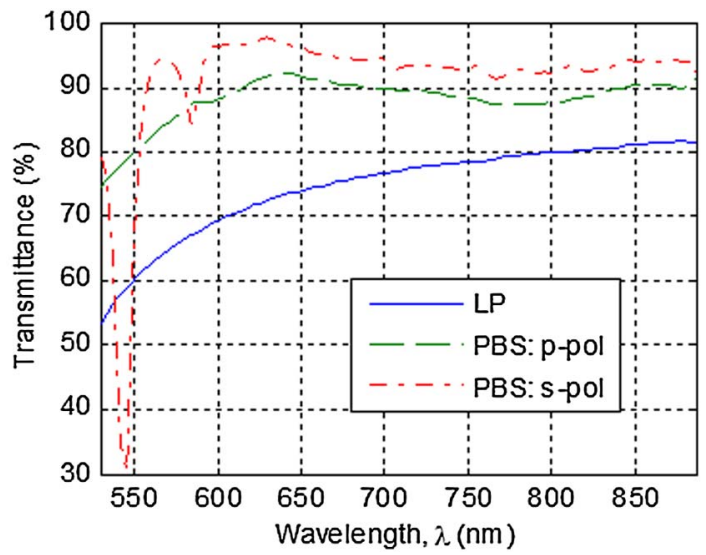

Fig. 4. (Color online) Experimental characterization of the linear polarized (LP), and polarization beam splitter (PBS) transmitted output ( $p$-pol) and reflected output ( $s$-pol), by using polarized light in the transmission axis direction.

in the first stage and two LC devices are used in the second stage; see Fig. 3. The same control volttage, $V_{\mathrm{LC}}\left(\mathrm{V}_{\mathrm{RMS}}\right)$, is applied to all LC cells.

Effective birefringence of the LC devices has been characterized in the range from 500 to $890 \mathrm{~nm}$ (limited by LPs and spectrometer operation range), and using $V_{\mathrm{LC}}$ values from 0 to $5 \mathrm{~V}_{\mathrm{RMS}}$, with steps of $5 \mathrm{~m} \mathrm{~V}_{\text {RMS }}$. Figure 5 (a) shows characterization results at three wavelengths. From full range characterization, an LC threshold voltage of $0.75 \mathrm{~V}_{\mathrm{RMS}}$ is obtained. LC device transmittance has been characterized in the range from 400 to $890 \mathrm{~nm}$; see Fig. 5(b). $1 \times 2$ WSS measurements and simulations are shown in Fig. 6, for a particular $V_{\mathrm{LC}}$ of $0.8 \mathrm{~V}_{\mathrm{RMS}}$ applied to all LCs. Transmission simulations are based on 
(a)
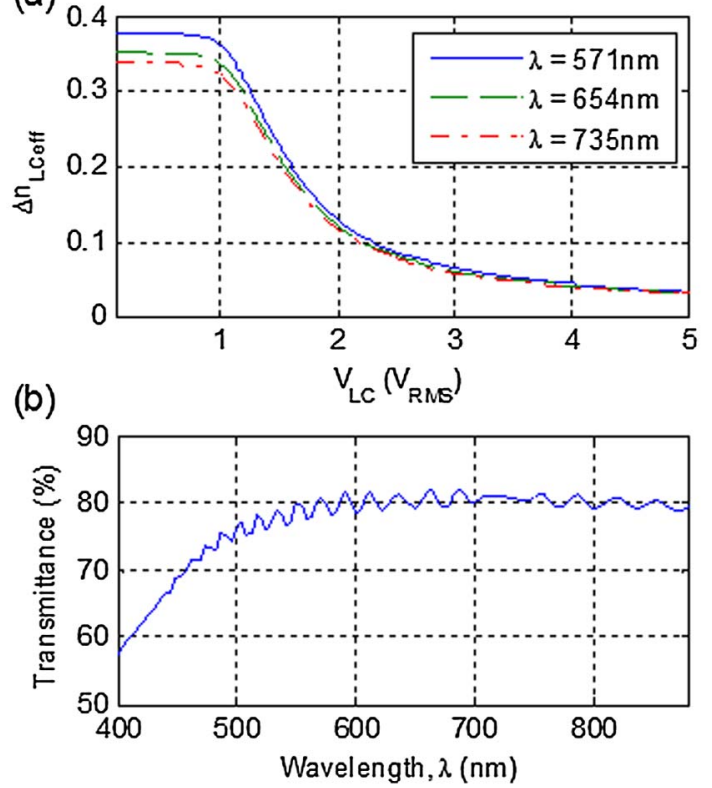

Fig. 5. (Color online) Characterization of the LC devices with mixture 1658: (a) effective birefringence, $\Delta n_{\mathrm{LC}_{\text {eff }}}$, as a function of the applied voltage, $V_{\mathrm{LC}}$, for three different wavelengths and (b) transmittance in the visible and near infrared spectrum.
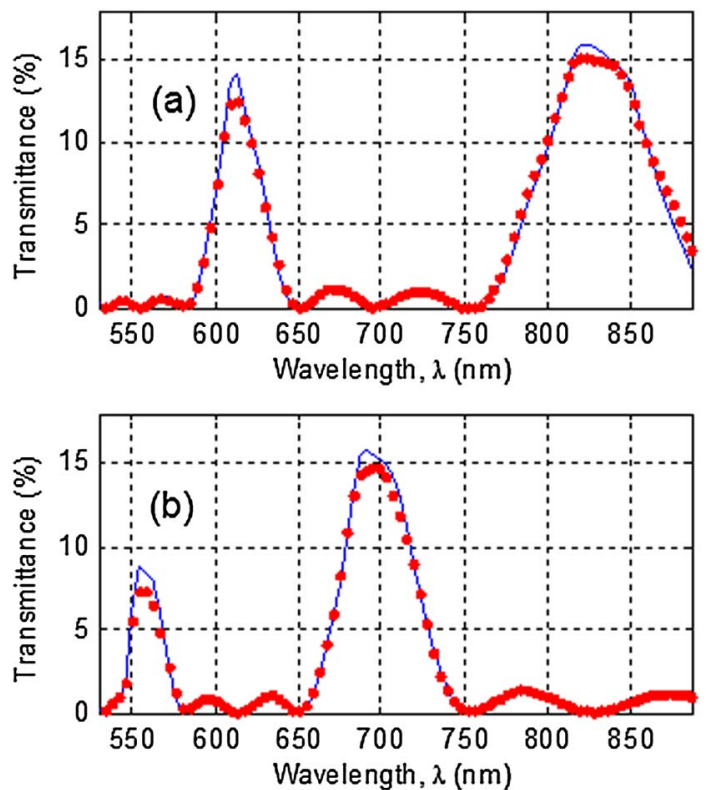

Fig. 6. (Color online) $1 \times 2$ WSS transmission versus wavelength, simulations (dotted lines) and experimental results (solid lines) $V_{\mathrm{LC}}=0.8 \mathrm{~V}_{\mathrm{RMS}}$ : (a) output $S_{1}$ and (b) output $S_{2}$.

Jones matrix transmission products reported elsewhere [20]. The simulation program designs each stage independently and takes into account all the filter parameters, such as the number of stages, the number of birefringent plates at each stage, tilt angles, losses of the components, and the birefringence of the LC material as well as its dispersion (in the characterized range). For getting accurate simulation results, experimental $\mathrm{LC}, \mathrm{LPs}$, and the PBS parameters previously reported were used.

From Fig. 6, it can be seen that there is a good agreement between simulations and experimental results. So, using the individual experimental characterization of WSS elements and the developed simulation program, transmission results for different $1 \times 2$ WSS configurations can be derived.

In the following, reconfigurable $1 \times 2 \mathrm{WSS}$ with polarization diversity in a POF network application is designed and simulated. This device (see Fig. 2) can demultiplex, switch, or block wavelength channels $\left(\mathrm{ch}_{1}\right.$ and $\left.\mathrm{ch}_{2}\right)$ between one input port and one or two output ports. Those transmission states are controlled by applying the appropriate $V_{\mathrm{LC}}$ voltage to all LC cells. Table 1 summarizes the eight possible transmission states of this $1 \times 2$ reconfigurable WSS.

LED-based channels at $560 \mathrm{~nm}\left(\mathrm{ch}_{1}\right)$ and $621 \mathrm{~nm}$ $\left(\mathrm{ch}_{2}\right)$ with full width half maximum (FWHM) of 42 and $18 \mathrm{~nm}$, respectively, are used as two channel inputs with typical POF network specifications [21]. $1 \times 2$ WSS is designed using all elements previously described in this section along with $98 \%$ reflectivity mirrors. An optimization algorithm, based in the characterization information of the LC devices, is used for determining the LC control voltages for reaching the different WSS transmission states. The following control voltages are obtained from this optimization process: $\left[V_{L C 1}, V_{L C 2}, \ldots, V_{L C 8}\right]=$ $[1.175,0.210,1.530,1.790,1.955,1.640,2.100,3.225]$ $\mathrm{V}_{\mathrm{RMS}}$. The voltage of $0.21 \mathrm{~V}_{\mathrm{RMS}}$ can be replaced by any voltage below $0.75 \mathrm{~V}_{\mathrm{RMS}}$ ( $V$ th). Simulations are shown in Fig. 7 for transmission states reported in Table 1. Impairments such as insertion loss, passband effects, and crosstalk are also calculated. They are given by the following:

Insertion losses (IL):

$$
\operatorname{IL}\left(\operatorname{ch}_{t}\right)_{S x}=-10 \log \left[\frac{P\left(\operatorname{ch}_{t}\right)_{S x}}{P\left(\operatorname{ch}_{t}\right)_{S o}}\right] \text { with }\left\{\begin{array}{l}
t=\{1,2\} \\
x=\{1,2\}
\end{array},\right.
$$

where $P\left(\operatorname{ch}_{t}\right)_{S x}$ and $P\left(\operatorname{ch}_{t}\right)_{S o}$ are optical power at the output $S_{x}$ and input $S_{O}$, respectively, for each wavelength channel, $\mathrm{ch}_{t}$. In these calculations, the whole spectrum of each channel is considered.

Rejection ratio (RR):

$$
\mathrm{RR}\left(\mathrm{ch}_{t}\right)_{S(x, y)}=-10 \log \left[\frac{P\left(\mathrm{ch}_{t}\right)_{S x}}{P\left(\mathrm{ch}_{t}\right)_{S y}}\right] \text { with }\left\{\begin{array}{l}
t=\{1,2\} \\
x=\{1,2\} \\
y=\{1,2\} \\
y \neq x
\end{array}\right.
$$

where $P\left(\operatorname{ch}_{t}\right)_{S x}$ and $P\left(\mathrm{ch}_{t}\right)_{S y}$ are optical power at desired output $S_{x}$ and opposite output $S_{y}$, respectively, for each wavelength channel, $\mathrm{ch}_{t}$. Output channel optical power is calculated at the maximum transmission.

Figure 7 shows that IL of 3.4 to $6 \mathrm{~dB}$ and RRs ranging from 12 to $15 \mathrm{~dB}$, in the different configurations, 


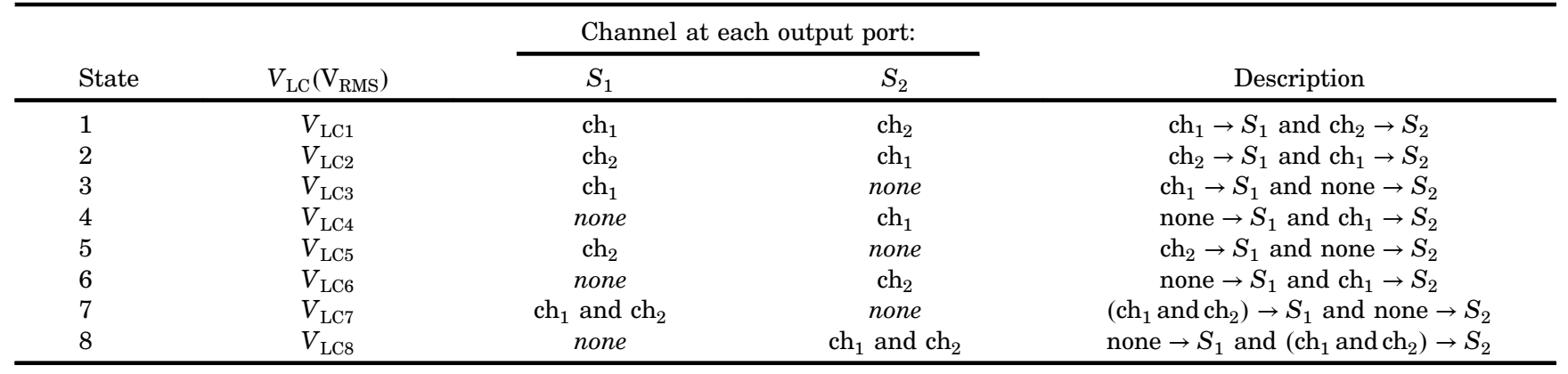

(a)

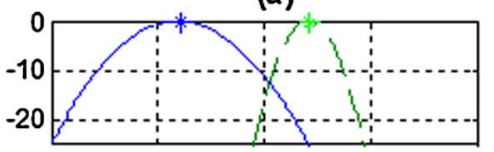

(b)

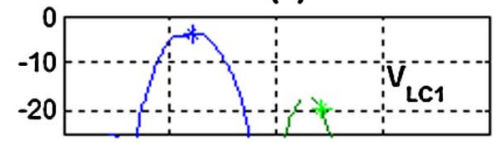

(c)
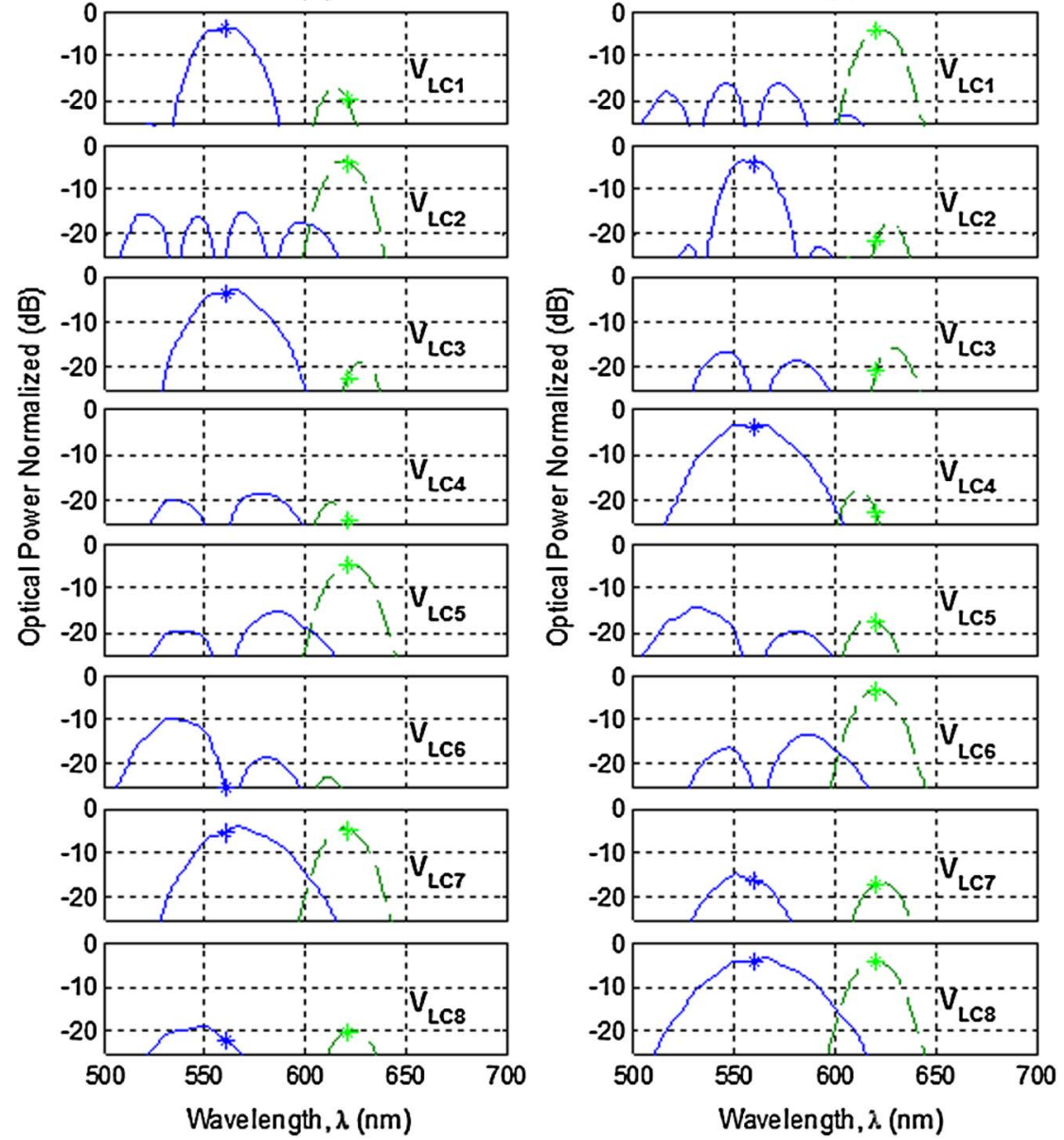

Fig. 7. (Color online) Normalized optical power of $1 \times 2$ WSS with polarization diversity versus wavelength in $\mathrm{ch}_{1}$ (solid line) and $\mathrm{ch}_{2}$ (dashed line) with $\left[V_{L C 1}, V_{L C 2}, \ldots, V_{L C 8}\right]=[1.175,0.210,1.530,1.790,1.955,1.640,2.100,3.225] \mathrm{V}_{\mathrm{RMS}}$. (a) $S_{0}$ input spectrum; (b) $S_{1}$ output spectrum; (c) $S_{2}$ output spectrum. Asterisks mark the central channel wavelengths.

are obtained. RR can be improved by adding more filtering stages to the $1 \times 2$ WSS design but at the expense of increasing IL. In a future work and after an optimization process, different voltage levels at each LC cell will be applied to improve RR among others.

\section{Conclusions}

A reconfigurable $1 \times 2 \mathrm{WSS}$, based on a Lyot filter structure, using specific high birefringence nematic LCs, has been designed and tested. The system working principle has been demonstrated in the visible spectrum region for plastic optical fiber networks. 
A versatile behavior for the $1 \times 2$ reconfigurable WSS is obtained by applying a variable voltage to each LC cell. Performance of a two channel system, at 560 and $621 \mathrm{~nm}$, is shown after optimizing LC voltages. This system allows demultiplexing, switching or blocking any channel through any output port using voltages from 0 to $3 \mathrm{~V}_{\mathrm{RMS}}$. Maximum IL of $6 \mathrm{~dB}$, and RRs better than $12 \mathrm{~dB}$, are obtained.

This work was partially supported by the Spanish CICYT (grant No. TEC2009-14718-C03-03) and CAM (grant No. S2009/ESP-1781). The authors acknowledge the Military University of Technology (MUT) in Warsaw for supplying the LC devices.

\section{References}

1. D. M. Marom, D. T. Neilson, D. S. Greywall, C.-S. Pai, N. R. Basavanhally, V. A. Aksyuk, D. O. Lopez, F. Pardo, M. E. Simon, Y. Low, P. Kolodner, and C. A. Bolle, "Wavelength-selective $1 \times K$ switches using free-space optics and MEMS micromirrors: theory, design, and implementation," J. Lightwave Technol. 23, 1620-1630 (2005).

2. J. S. Patel and Y. Silberberg, "Liquid crystal and grating-based multiple-wavelength cross-connect switch," IEEE Photon. Technol. Lett. 7, 514-516 (1995).

3. B. Fracasso, J. L. de Bougrenet de la Tocnaye, M. Razzak, and C. Uche, "Design and performance of a versatile holographic liquid crystal wavelength selective optical switch," J. Lightwave Technol. 21, 2405-2411 (2003).

4. C. Vázquez, I. Pérez, P. Contreras, B. Vinouze, and B. Fracasso, "Liquid crystal optical switches," in Optical Switches: Materials and Design, B. Li and S. J. Chua, eds. (Woodhead, 2010), Chap. 8.

5. K. Suzuki, T. Mizuno, M. Oguma, T. Shibata, H. Takahashi, Y. Hibino, and A. Himeno, "Low loss fully reconfigurable wavelength-selective optical $1 \times N$ switch based on transversal filter configuration using silica-based planar lightwave circuit," IEEE Photon. Technol. Lett. 16, 1480-1482 (2004).

6. C. Vázquez, P. C. Lallana, J. Montalvo, J. M. Sanchez Pena, A. d'Alessandro, and D. Donisi, "Switches and tunable filters based on ring resonators and liquid crystals," Proc. SPIE $6593,65931 \mathrm{~F}$ (2005)

7. K. Vlachos, C. Raffaelli, S. Aleksic, N. Andriolli, D. Apostolopoulos, H. Avramopoulos, D. Erasme, D. Klonidis, M. N. Petersen, M. Scaffardi, K. Schulze, M. Spiropoulou, S. Sygletos, I. Tomkos, C. Vázquez, O. Zouraraki, and F. Neri,
"Photonics in switching: enabling technologies and subsystem design,” J. Opt. Netw. 8, 404-428 (2009).

8. S. Cao, J. Chen, J. N. Damask, C. R. Doerr, L. Guiziou, G. Harvey, Y. Hibino, H. Li, S. Suzuki, K.-Y. Wu, and P. Xie, "Interleaver technology: comparisons and applications requirements," J. Lightwave Technol. 22, 281-289 (2004).

9. B. Lyot, "Optical apparatus with wide field using interference of polarized light," C. R. Acad. Sci. (Paris) 197, 1593 (1933).

10. Y. Ohman, "A new monochromator," Nature 141, 157-158 (1938).

11. M. Huang, J. Chen, J. Yu, S. Chi, and G. Chang, "A novel dispersion-free interleaver for bidirectional DWDM transmission systems," J. Lightwave Technol. 25, 3543-3554 (2007).

12. C. Lee, R. Wang, P. Yeh, and W. Cheng, "A new scheme of birefringent optical interleaver employing ring cavity as phase-dispersion element," in Conference on Lasers and Electro-Optics / Quantum Electronics and Laser Science Conference and Photonic Applications Systems Technologies Technical Digest (CD) (Optical Society of America, 2007), paper JTuA30.

13. D. Visani, Y. Shi, C. M. Okonkwo, H. Yang, H. P. A. van den Boom, G. Tartarini, E. Tangdiongga, and A. M. J. Koonen, "Wired and wireless multi-service transmission over 1mm-core GI-POF for in-home networks," Electron. Lett. 47, 203-205 (2011).

14. J. Vinogradov, O. Ziemann, and R. Kruglov, "Data transmission with blue laser diodes," in POF-PLUS Handbook, O. Ziemann, ed. (POF-AC Nürnberg, 2011), p. 51.

15. M. Joncic, M. Haupt, and U. H. P. Fischer, "Spectral grids for VIS WDM applications over SI-POF," presented at 20th International Conference on Plastic Optical Fibers, Bilao, Spain, 14-16 September 2011, paper STD-130.

16. O. Aharon and I. Abdulhalim, "Liquid crystal Lyot tunable filter with extended free spectral range," Opt. Express 17, 11426-111433 (2009).

17. P. C. Lallana, C. Vázquez, and B. Binouze, "Advanced multifunctional optical switch for multimode optical fiber networks," Opt. Commun. 285, 2802-2808 (2012).

18. J. Li, C. Wen, S. Gauza, R. Lu, and S. Wu, "Refractive indices of liquid crystals for display applications," J. Disp. Technol. 1, 51-61 (2005).

19. Refractive Index Database, http://refractiveindex.info/? group=LC\&material=MLC-9200-100 (2012).

20. P. J. Pinzón, I. Pérez, V. Urruchi, C. Vázquez, and J. M. Sanchez Pena, "Tunable optical filter using high birefringence nematic liquid crystals," presented at OPTOEL'11, Santander, Spain, 29 June-1 July 2011, paper S3-111.

21. O. Ziemann, J. Krauser, P. E. Zamzow, and W. Daum, POF Handbook: Optical Short Range Transmission Systems, 2nd ed. (Springer, 2008). 